(C) 2010 Elsevier B.V. All rights reserved. Access to this work was provided by the University of Maryland, Baltimore County (UMBC) ScholarWorks@UMBC digital repository on the Maryland Shared Open Access (MD-SOAR) platform.

Please provide feedback

Please support the ScholarWorks@UMBC repository by emailing scholarworks-group@umbc.edu and telling us what having access to this work means to you and why it's important to you. Thank you. 


\section{THE UNCOVERED SET AND INDIFFERENCE IN SPATIAL MODELS: \\ A FUZZY SET APPROACH}

\section{Cycling in Fuzzy Spatial Models}

In spite of substantial criticism [1], a great number of spatial models are grounded in the rational choice assumption that political actors are self-interested, utility maximizers possessing Euclidean preferences over alternatives. Were it not for the substantial incongruence between the predictions made by these models and empirical reality, criticism of the assumptions behind the models might be more easily dismissed. The most well-known incongruence is the majority cycling problem. McKelvey [2] found that in the absence of Plott's [3] radial symmetry, outcomes of majority rule games under assumptions of sincere voting should lead anywhere. The problem is that there is little empirical evidence of cycling in political life [4], a fact that has led many to question the validity of spatial models.

In more formal terms, McKelvey argues that there is no core, or set of majority preferred outcomes. A core is defined as

$$
M(R, X)=\{x \in X \mid \forall y \in X, \sim y P x\}
$$

where $X$ is the set of alternatives, $R$ is a binary relation, and $R$ is complete. $P$ is the strict preference relation associated with $R$. Let $N$ denote the set of players 
and let $n$ denote the number of players. Let $\mathbb{R}$ denote the set of all binary relations on $X$ that are reflexive, complete, and transitive. Let $\mathbb{R}^{n}$ denote the set of all $\mathrm{n}$ tuples $\rho=\left(R_{1}, \ldots, R_{n}\right)$, where $R_{i}$ belongs to $\mathbb{R}, i=1, \ldots, n$. Let $f$ be a majority aggregation rule on $\mathbb{R}^{n}$. We suppress the notation $f(\rho)$ and simply write $R$. Then $P$ denotes the strict preference relation associated with $f(\rho)$.

We will refer to a core as a maximal set under majority rule. We define majority rule as

$$
x P y \equiv\left|\left\{i \mid x P_{i} y\right\}\right|>\frac{n}{2}
$$

If $x$ defeats $y$ under majority rule, then $x P y \equiv\left|\left\{i \mid x P_{i} y\right\}\right|>\frac{n}{2}>\left|\left\{j \mid y P_{j} x\right\}\right|$. In the absence of a majority rule maximal set, there is no equilibrium, majority cycling results, and spatial models can not predict outcomes.

An approach to resolving the majority cycling problem, that has been long known, but remains largely under-developed, is the introduction of thick indifference to models [5]. Several studies have found that the probability of a majority rule maximal set increases when actors are indifferent over regions of the policy space $[6,7,8]$. Much of this work makes use of the epsilon-core. Until the distance between two alternatives exceeds some arbitrary distance $\epsilon$, actors are indifferent in a choice between them $[9,10]$. Actors are essentially indifferent to alternatives within radius $\epsilon$ from one another, in a region defined as the $\epsilon$-core.

A recent addition to the literature on indifference offers a fuzzy set theory ap- 
proach to spatial models [11]. The fuzzy approach not only permits the modeling of a substantial degree of indifference, it also thickens the line that defines a player's win-circle. Furthermore, in contrast to the $\epsilon$-core concept, it models uniform indifference over a fixed and discrete region of the policy space. Nonetheless, despite predicting a majority rule maximal set under a considerable number of conditions, fuzzy spatial models do not guarantee a stable maximal rule outcome in all cases [11]. This raises the issue of how to arrive at a prediction set when spatial models do not generate a maximal set.

One strand of the public choice literature argues that, in the absence of a majority rule maximal set, political actors should choose an alternative in the uncovered set, the set of alternatives that sophisticated voters would reach by some amendment agenda $[12,13,14]$. The specific alternative chosen depends on the amendment order. This paper develops a fuzzy uncovered set. We follow the lead in [11] and adopt discrete fuzzy numbers to represent the preferences of

political actors. We demonstrate that with one relatively mild exception, the fuzzy uncovered set comprises an improper subset of the Pareto set.

\section{Fuzzy Spatial Models}

Fuzzy set spatial models permit us to model a substantial degree of indifference in political actors' preferences over policy options. Each element $x$ in the universe of policy alternatives $X$ over which political players are making choices are assigned 
a value on the interval $[0,1]$. The assigned value indicates the degree to which a political actor considers the given alternative to be an ideal policy. Formally, each element $x$ has a degree of set inclusion, $\widetilde{F}(x)$, in the set of ideal preferences that is specified by a function $\widetilde{F}[15]$. For $\alpha$ in $[0,1]$, the sets $F^{\alpha}=\{x \in X \mid F(x) \geq \alpha\}$ are referred to as alpha-levels ( $\alpha$-levels).

Individual indifference is captured by the $\alpha$-level concept. A political player is indifferent between all alternatives at the same $\alpha$-level, essentially treating them as equivalents. Alpha-levels may take any value, which permits modelers to distinguish between actors who are more or less discerning in their preferences. The conventional approach locks us into modeling players who have the capacity to make infinitesimally small distinctions between alternatives. While a fuzzy approach can accommodate such an assumption by assigning $\alpha$-levels along the continuum from 0 to 1 , individual preferences over policies are likely to be substantially less discriminating. Following the lead in [11], we model such coarsegrained distinctions using a finite set of discrete $\alpha$-levels $T=\{0, .25, .5, .75,1\}$. This Likert-like scale has an intuitive appeal to it. The set of ideal policy preferences for an actor are assigned an $\alpha$-level of 1 (full membership in the set of ideal policies). All remaining policies are scored on the degree to which they come close to being ideal. Those considered almost ideal are assigned .75 (three-fourths membership in the set of ideal policies), those considered "neither ideal nor not ideal" are scored .50 (one-half membership in the set of ideal policies), those "less 
ideal than ideal" are scored .25 (one-quarter membership in the set of ideal policies), and those that are "not ideal" to any degree 0 (no membership in the set of ideal policies).

Since all alternatives at the same $\alpha$-level are equally preferred to one another, a player is indifferent to all alternatives at a given $\alpha$-level. Thus, fuzzy spatial models map individual preferences as bounded regions (defined by each $\alpha$-level), within which a political player can not differentiate among policy positions.

Consider figure 1, which maps the preferences of three players $N=\{A, B, C\}$ at $\alpha=1, .75, .50$, and .25 in two-dimensional space. The inner-most regions represent $\alpha=1$. The intersection of the $\alpha$-levels for the three players (threetuples) are noted in parentheses, $(\widetilde{A}(x), \widetilde{B}(X), \widetilde{C}(x))$. If a maximal set exists under majority rule, it falls in one of the intersections of a majority of players' $\alpha$-levels. The set of options that are majority preferred (the winset) to each numbered alternative are noted. All alternatives are majority preferred by at least one other alternative. Hence, there is no majority rule maximal set in the situation defined by these sets of preferences.

[Place Figure 1 here.]

The potential for cycling in fuzzy spatial models leaves open the question of how to predict outcomes in the absence of a majority rule maximal set. This paper develops a fuzzy uncovered set as an alternative prediction set when no majority 
rule maximal set exists. In what follows, we demonstrate that with one exception our fuzzy uncovered set is an improper subset of the Pareto set (Theorem 10). As it turns out, the exception is quite mild, since it requires that no alternative is majority preferred to any other.

\section{A Definition of Covering Given Fuzzy Preferences}

The uncovered set is the set of sophisticated voting outcomes in an amendment agenda. Miller [12] originally examined the covering relation and the uncovered set in majority preference tournaments (amendment agendas) that result when an odd number of voters have strong preferences over discrete alternatives. Formally, the uncovered set is defined as

$$
U C(X)=\{x \in X \mid \sim y C x \forall y \in X\}
$$

where $C$ is a covering relation. Provided that $C$ is defined in a manner that makes it both asymmetric and acyclic (as in the tournament case), $U C(X)$ is never empty. Miller $[12,16]$ originally assumed that players would exercise strict preference over all alternatives. He defined he covering relation is defined as

$$
{ }_{x} C y \equiv W^{-1}(y) \subseteq W^{-1}(x),
$$

where $W^{-1}(x)$ is the set of all alternatives to which $x$ is strictly preferred by a

majority. $W^{-1}(x)$ is everything that $x$ beats. $W^{-1}(x)$ is called the inverse winset 
of $x$. Formally, $W^{-1}(x)=\{y \in X \mid x P y\}$. In words, $x$ covers $y$ if and only if every point beaten by $y$ is also beaten by $x$.

A number of alternative definitions of the covering relations $C$ have subsequently been proposed. In the conventional approach to spatial modeling, indifference among an odd number of players is "thin." (Indifference is limited to the indifference curves that describe individual preference.) Under conditions of thin indifference, all of the definitions of the covering relation in the literature are equivalent. However, Penn [17] and Miller [18] have noted that in any context that allows ties, these equivalences break down,

In the case of fuzzy spatial models, both individual indifference and collective indifference (tie sets) are "thick." Hence, the equivalence relations among the various proposed definitions of the covering relation do not hold; and the definitions of the covering relation return differing results $[17,19]$.

Several types of collective indifference are possible in the fuzzy approach. Consider the three-player case. The three-tuple representing the alternative lying at the intersection of the preferences $(\alpha$-levels) of three players at $(.75, .5, .25)$ is not majority preferred to that at $(.75, .5, .0)$. In this case, players one and two would select either alternative since each of the players in the coalition views the policies as essentially the same (in terms of their respective preferences). The second type of indifference that can occur is when the same coalition members prefer two different points, but each member of the coalition prefers the point at a different 
$\alpha$-level. Take for instance, $(.75, .5, .25)$ and $(.5, .75, .25)$. Here we have a case in which the same coalition is indifferent between two alternatives, but player one prefers the first to the second, but player two prefers the second to the first. A third type of indifference occurs when a single player has the ability to form more than one coalition that would allow her to choose between alternatives that she prefers at the same $\alpha$-level. Consider $(.75, .5, .25)$ and $(.25, .5, .75)$. Player two has the option of forming a coalition with either player one or three. It is impossible to know the choice she will make since she is indifferent between the two alternatives. A fourth type of indifference is more easily resolvable. Suppose players one and two have a choice between $(.75, .5, .0)$ and $(.75, .25, .0)$. While the two-player coalition can choose either over the objections of the third player, if player two is willing to hold out, the first alternative will be selected.

Collective indifference leaves open the question of an appropriate definition of the covering relation given fuzzy preferences. Thus, we must enter unexplored territory to come with a definition of covering that seems most appropriate in the fuzzy context.

In what follows, we identify an appropriate definition for the covering relationship when indifference over alternatives is introduced. We proceed by reconsidering the set of plausible outcomes in sophisticated voting agenda. We conclude by formally characterizing the fuzzy uncovered set. We are guided in our task by Miller [12] and Shepsle and Weingast [13]. The latter formalize a process for 
determining the sophisticated outcome in an amendment agenda. Their process focuses on levels in the voting order rather than the decision nodes in the voting tree representing an amendment agenda. Formally, a voting tree is "an amendment agenda if there exists an ordering $\alpha:\{1, \ldots, r\} \rightarrow X$ such that the majority voting sequence is $(\alpha(1), \alpha(2), \ldots, \alpha(r))$, where $\alpha(1)$ is first voted against $\alpha(2)$, the winner against $\alpha(3)$, etc." (Austen-Smith and Banks 2005, p. 132).

We demonstrate Shepsle and Weingast's approach as follows. Suppose that we have a cycle set: $(.25, .25, .5) P(.75,0, .25) P(.5,1,0) P(.25, .75,1) P(1, .5, .75)$. Let the agenda order $A$ be:

$$
A=\{(.25, .25, .5),(1, .5, .75),(.5,1,0),(.25, .75,1),(.75,0, .25)\}
$$

Any alternative chosen by sophisticated players must be preferred by a majority to the final alternative in the voting order. Those that fail to do so, can not be the sophisticated outcome. Furthermore, the sophisticated outcome must be majority preferred to all alternatives that are majority preferred at any level of the game. Following the Shepsle and Weingast procedure, the step-by-step results are: 


$$
\begin{aligned}
& \alpha(5)=(.75,0, .25) \\
& \alpha(4)=(.25, .75,1),(.25, .75,1) P(.75,0, .25) \\
& \alpha(3)=(.25, .75,1),(.5, .1,0) P(.25,75,1) \text { but }(.75,0, .25) P(.5,1,0) \\
& \alpha(2)=(.25, .75,1),(1, .5, .75) P(.75,0, .25) \text { but }(.25, .75,1) P(1, .5, .75) \\
& \alpha(1)=(.25, .75,1),(.25, .25, .5) P(.75,0, .25) \text { but }(.25, .75,1) P(.25, .25, .5)
\end{aligned}
$$

By reflexivity, the final alternative is at least as good as itself. Hence, in the construction of the backward induction process that is common to determining the sophisticated winner in a voting game, $(.75,0, .25)$ is trivially the winner at the level $\alpha(5)$. At the next level, $(.25, .75,1)$ is majority preferred to $(.75,0, .25)$. Hence, it is the alternative at level $\alpha(4)$. While $(.5,1,0)$ defeats $(.25, .75,1)$ at level $\alpha(4)$, it is defeated by $(.75,0, .25)$ at level $\alpha(5)$. Thus, $(.25, .75,1)$ carries over as the winner at level $\alpha(3)$. By the same reasoning, $(.25, .75,1)$ carries over as the winner at levels $\alpha(2)$ and $\alpha(1)$. Since it is the winner at level $\alpha(1)$, it is the sophisticated majority winner.

While this particular voting order appears to suggest that Shepsle and Weingast's [13] method can be used with fuzzy preferences, the problem induced by indifference comes into full view if we consider the voting agenda

$$
A=\{(.75, .25,0),(.25,0, .75),(0, .5, .75),(.75,0, .5),(.75, .5,0)\}
$$

The results through the first three levels are: 


$$
\begin{aligned}
& \alpha(5)=(.75, .5,0) \\
& \alpha(4)=\text { outcome uncertain },(.75, .5,0) I(.75,0, .5) \\
& \alpha(3)=\text { outcome uncertain, }(0, .5, .75,) P(.75, .0, .5) \text { but }(0, .5, .75) I(.75, .5,0)
\end{aligned}
$$

In this case, $(.75, .5,0)$ is trivially majority preferred at level $\alpha(5)$, but it is tied (it is indifferent, as noted by $I$ ) with $(.75,0, .5)$ at level $\alpha(4)$. Furthermore, while $(0, .5, .75)$ defeats $(.75,0, .5)$ by a majority, it is indifferent to $(.75, .5,0)$.

We are faced with two issues: (1) how to proceed when an alternative defeats at least one alternative majority preferred at previous levels but ties all others and (2) how to proceed when an alternative ties all alternatives majority preferred at previous levels? Issue (1) is the easiest of the two challenges to deal with. An alternative that defeats at least one alternative more than a successor in the voting order is superior to the successor. Therefore, we should designate it the winner at the given level. However, in the case of issue (2), since neither alternative is superior in this sense, we are compelled to accept having to list both at the given level. This forces us to compare subsequent predecessors with both alternatives. The guiding principle is that the sophisticated outcome is one that majority defeats one or more majority preferred successor and ties all others.

Using this procedure, we get, 


$$
\begin{aligned}
& \alpha(5)=(.75, .5,0) \\
& \alpha(4)=(.75, .5,0) \&(.75,0, .5),(.75, .5,0) I(.75,0, .5) \\
& \alpha(3)=(0, .5, .75),(0, .5, .75,) P(.75,0, .5) \text { and }(0, .5, .75) I(.75, .5,0) \\
& \alpha(2)=(0, .5, .75),(.25,0, .75) I(0, .5, .75),(.25,0, .75) I(.75,0, .5), \text { but }(.75, .5,0) P(.25,0, .75) \\
& \alpha(1)=(0, .5, .75),(.75, .25,0) I(.75, .5,0),(.75, .25,0) I(.75,0, .5), \text { but }(0, .5, .75) P(.75, .25,0)
\end{aligned}
$$

The alternative represented by the preference three-tuple $(0, .5, .75)$ is the sophisticated outcome given this amendment agenda, and it is an element in the uncovered set.

The procedure that we followed is defined by the following definition of covering,

$$
x C y \Longleftrightarrow x R y \& W^{-1}(y) \subset W^{-1}(x) \Longrightarrow x C_{3}^{\prime} y \&^{\sim} y C_{3}^{\prime} x .
$$

In words, $x$ is at least tied with $y, x$ beats everything that $y$ beats, and $x$ beats something (perhaps $y$ itself) that $y$ fails to beat. Bordes [20] labels the uncovered set that results from this definition the FD set. It differs from any of the three definitions considered by Penn [17], all of which imply that $x$ is strictly preferred to $y, x P y$. Henceforth, we use this definition of covering, which we formally define in Definition 10.

In what follows, we give formal consideration to $U C(X)$. Our major theorem, Theorem 10, is that with one mild exception, any element in $U C(X)$ is in the 
Pareto set. Formally, the Pareto set is $[21$, p. 7]

$$
P S_{N}(R)=\{x \in X \mid \forall y \neq x, P(y, x ; R) \neq \emptyset \Longrightarrow P(x, y ; R) \neq \emptyset\}
$$

\section{The Uncovered Set with Fuzzy Preferences}

Let $N$ denote the set of players and $X$ denote the set of alternatives. We assume that $X$ is a subset of a universe $U$ of interest. Let $\mathcal{R}$ denote the set of all binary relations on $X$ which are reflexive, complete and transitive. Let $\mathcal{R}^{n}=\left\{\boldsymbol{\rho} \mid \boldsymbol{\rho}=\left(R_{1}, \ldots, R_{n}\right), R_{i} \in \mathcal{R}, i=1, \ldots, n\right\}$, where $|N|=n$. Let $\leq$ be a partial order on $U$. Suppose that $\leq$ satisfies the following properties:

(1) $\forall x, y \in U, x \leq y$ implies $\forall i \in N, y R_{i} x$;

(2) $\forall x, y, z \in U, \forall i \in N, x \leq y$ and $x R_{i} z$ implies $y R_{i} z$;

(3) $\forall x, y, z \in U, \forall i \in N, x \leq y$ and $x P_{i} z$ implies $y P_{i} z$;

(4) $\forall x, y \in U, x<y$ implies $\exists i \in N$ such that $y P_{i} x$;

(5) $\forall x, y, z \in U, \forall i \in N, x \leq y$ and $z R_{i} y$ implies $z R_{i} x$;

(6) $\forall x, y \in U, x$ and $y$ incomparable under $\leq$ implies $\exists i \in N$ such that $x P_{i} y$ $\operatorname{implies} \exists j \in N$ such that $y P_{j} x$.

Definition 1 Define \langle\rangle$: \mathcal{P}(U) \rightarrow \mathcal{P}(U)$ by $\forall S \in \mathcal{P}(U),\langle S\rangle=\{x \in U \mid \exists s \in$ $S, x \leq s\}$

Proposition 2 Let \langle\rangle$: \mathcal{P}(U) \rightarrow \mathcal{P}(U)$ be defined as above. Then the following conditions hold. 
1. $\forall S \in \mathcal{P}(U), S \subseteq\langle S\rangle$;

2. $\forall S_{1}, S_{2} \in \mathcal{P}(U), S_{1} \subseteq S_{2}$ implies $\left\langle S_{1}\right\rangle \subseteq\left\langle S_{2}\right\rangle$;

3. $\forall S \in \mathcal{P}(U),\langle S\rangle=\langle\langle S\rangle\rangle$;

4. $\forall S \in \mathcal{P}(U),\langle S\rangle=\cup_{s \in S}\langle\{s\}\rangle ;$

5. $\forall S \in \mathcal{P}(U), \forall x, y \in X, x \in\langle S \cup\{y\}\rangle$ and $x \notin\langle S\rangle$ implies $x \in\langle\{y\}\rangle$.

Proof. (1) Let $s \in S$. Then $s \leq s$ and so $s \in\langle S\rangle$. Thus $S \subseteq\langle S\rangle$.

(2) Let $x \in\left\langle S_{1}\right\rangle$. Then there exists $s \in S_{1}$ such that $x \leq s$. Since $s \in S_{2}, x \in$ $\left\langle S_{2}\right\rangle$.

(3) By $(1),\langle S\rangle \subseteq\langle\langle S\rangle\rangle$. Let $x \in\langle\langle S\rangle\rangle$. Then there exists $y \in\langle S\rangle$ such that $x \leq y$. There exists $s \in S$ such that $y \leq s$. Since $\leq$ is transitive, $x \leq s$. Thus $x \in\langle S\rangle$. Hence $\langle\langle S\rangle\rangle \subseteq\langle S\rangle$.

(4) For all $s \in S,\langle\{s\}\rangle \subseteq\langle S\rangle$ by (2). Thus $\cup_{s \in S}\langle\{s\}\rangle \subseteq\langle S\rangle$. Let $x \in\langle S\rangle$. Then there exists $s \in S$ such that $x \leq s$. Thus $x \in\langle\{s\}\rangle$ and so $x \in \cup_{s \in S}\langle\{s\}\rangle$. Hence $\langle S\rangle \subseteq \cup_{s \in S}\langle\{s\}\rangle$

(5) Suppose $x \in\langle S \cup\{y\}\rangle$ and $x \notin\langle S\rangle$. Then there does not exist $s \in S$ such that $x \leq s$. Hence $x \leq y$. Thus $x \in\langle\{y\}\rangle$.

Definition $3 M_{R}=\{x \in X \mid \nexists y \in X, x<y\}$.

Proposition $4 M_{R}=P S_{N}(R)$. 
Proof. Suppose $x \in M_{R}$. Let $y \in X$. Suppose $\exists i \in N$ such that $y P_{i} x$. Now there does not exist $y \in X$ such that $x<y$. Thus $\forall y \in X$, either $y \leq x$ or $x$ and $y$ are not comparable. Since $y P_{i} x, y \leq x$ is impossible else $x R_{i} y \forall i \in N$ by (1). Hence $x$ and $y$ are incomparable under $\leq$. Thus $\exists j \in N$ such that $x P_{j} y$ by (5). Hence $x \in P S_{N}(R)$. Thus $M_{R} \subseteq P S_{N}(R)$.

Suppose $x \in P S_{N}(R)$. Suppose there exists $y \in X$ such that $x<y$. Then $\exists i \in N$ such that $y P_{i} x$. Since $x \in P S_{N}(R)$, there exists $j \in N$ such that $x P_{j} y$. Thus $x<y$ is impossible. Hence $x \in M_{R}$. Therefore $P S_{N}(R) \subseteq M_{R}$.

Corollary 5 Let $x \in X$.

(1) Suppose $\forall y \in X, x \leq y$ implies $x=y$. Then $x \in P S_{N}(R)$.

(2) If $x \notin P S_{N}(R)$, then there exists $y \in P S_{N}(R)$ such that $x<y$.

Proof. (1) Clearly $x \in M_{R}$, but $M_{R}=P S_{N}(R)$.

(2) Since $x \notin P S_{N}(R), x \notin M_{R}$. Thus there exists $y \in X$ such that $x<y$. Let $y$ be the largest such element. Then $y \in M_{R}=R S_{N}(R)$.

Proposition $6\langle X\rangle=\left\langle P S_{N}(R)\right\rangle$.

Proof. Clearly, $P S_{N}(R) \subseteq X$. Thus $\left\langle P S_{N}(R)\right\rangle \subseteq\langle X\rangle$. Let $x \in X$. If $x \notin$ $\left\langle P S_{N}(R)\right\rangle$, then $x \notin P S_{N}(R)$ and so by (2) of Corollary 5, there exists $y \in$ $P S_{N}(R)$ such that $x<y$. Thus $x \in\langle\{y\}\rangle \subseteq\left\langle P S_{N}(R)\right\rangle$. Hence $X \subseteq\left\langle P S_{N}(R)\right\rangle$ and so $\langle X\rangle \subseteq\left\langle P S_{N}(R)\right\rangle$.

Unless otherwise stated, we assume that $\left\langle P S_{N}(R)\right\rangle$ denotes the set of alter- 
natives. In other words, there is an alternative for every possible $n$-tuple of preferences descending from the Pareto set.

Let $T=\{0, .25, .5, .75,1\}$. Let $X \subseteq T^{n}$ and let $J_{\frac{n}{2}}=\left\{\widetilde{a} \in T^{n} \mid a_{i} \in\{0,1\}, i=\right.$ $1, \ldots, n,\}\left|\left\{i \mid a_{i}=0\right\}\right| \geq n / 2$ and $\exists j \in N$ such that $\left.a_{i}=1\right\} . \quad$ In the following result we use the notation [[]] to denote the greatest integer function.

Proposition 7 Let $x, y \in X$. If $x C y$, then either $x>y$ or $y \in\left\langle J_{\frac{n}{2}}\right\rangle$.

Proof. Suppose $x \leq y$. Then clearly $\nexists w \in X$ such that $x P w$ and not $y P w$, contradiction since $x C y$. Thus either $x>y$ or $x$ and $y$ are not comparable with respect to $\leq$. Suppose $x$ and $y$ are not comparable w. r. t. $\leq$. Then $\exists i, j \in N$ such that $x_{i}>y_{i}$ and $x_{j}<y_{j}$. Since $x C y, x R y$. Thus not $y P x$. Hence strictly fewer that $\left[\left[\frac{n}{2}\right]\right]+1$ of the $y_{i}$ are strictly greater then the corresponding $x_{i}$. There is no loss in generality in assuming $y_{1} \leq x_{1}, \ldots, y_{r} \leq x_{r}$ and $y_{r+1}>x_{r+1}, \ldots, y_{n}>x_{n}$ where $n-r<\left[\left[\frac{n}{2}\right]\right]+1$. Suppose $y_{1}=\ldots=y_{s-1}=0$ for $s \geq 1$. (The case $s=1$ says no $y_{i}=0$.) We show $s-1 \geq n / 2$. Assume $s-1<n / 2$. Then $n-s+1>n / 2$. Let $x=\left(x_{1}, \ldots, x_{n}\right)$ and $y=\left(y_{1}, \ldots, y_{n}\right)$. Let $t$ be such that $r-t-s+1+n-r=\left[\left[\frac{n}{2}\right]\right]+1$. Now let $y_{s}^{\prime}, \ldots, y_{r-t}^{\prime}$ be such that $y_{s}>y_{s}^{\prime} \geq 0, \ldots, y_{r-t}>y_{r-t}^{\prime} \geq 0$. Let

$$
z=\left(x_{1}, \ldots, x_{s-1}, \stackrel{r-t-s+1}{\prime} y_{s}^{\prime}, \ldots, y_{r-t}^{\prime}, x_{r-t+1}, \ldots, x_{r}, x_{r+1}, \ldots, x_{n}\right) .
$$


Now $n-t-s+1=\left[\left[\frac{n}{2}\right]\right]+1$. Thus

$$
t+s-1=\left\{\begin{array}{c}
{\left[\left[\frac{n}{2}\right]\right]-1 \text { if } n \text { is even }} \\
{\left[\left[\frac{n}{2}\right]\right] \text { if } n \text { is odd. }}
\end{array}\right.
$$

Now $t+s-1$ is the number of components $x_{i}$ is strictly greater than the corresponding components of $z$. Thus not $x P z$. However, $r-t-s+1+n-r=\left[\left[\frac{n}{2}\right]\right]+1$ (see above) is the number of components of $y$ that are strictly greater than the corresponding components of $z$. Thus $y P z$, contradicting the hypothesis that $x C y$. Thus $s-1 \geq n / 2$. Hence $y \in\left\langle J_{\frac{n}{2}}\right\rangle$.

Proposition 8 Let $x, y \in X$. If $x>y$, then either $(\exists w \in X$ such that $x P w$ and not $y P w)$ or $x \in\left\langle J_{\frac{n}{2}}\right\rangle$.

Proof. Suppose $x$ and $y$ differ in $\left[\left[\frac{n}{2}\right]\right]+1$ or more components, where [[]] denotes the greatest integer function. Then let $w=y$. Suppose $x$ and $y$ differ in fewer than $\left[\left[\frac{n}{2}\right]\right]+1$ components. There is no loss in generality in assuming that $x=\left(x_{1}, \ldots, x_{n}\right)$ and $y=\left(y_{1}, \ldots, y_{n}\right)$, where $x_{1}=y_{1}, \ldots, x_{r}=y_{r}, x_{r+1}>y_{r+1}, \ldots, x_{n}>y_{n}$, and $n-r \leq\left[\left[\frac{n}{2}\right]\right]$. Thus

$$
r \geq n-\left[\left[\frac{n}{2}\right]\right]=\left\{\begin{array}{c}
\frac{n}{2} \text { if } n \text { is even } \\
{\left[\left[\frac{n}{2}\right]\right]+1 \text { if } n \text { is odd }}
\end{array}\right.
$$

Suppose $x \notin\left\langle J_{\frac{n}{2}}\right\rangle$. Then fewer than $\frac{n}{2}$ of the $x_{i}=0$, say $x_{1}=\ldots=x_{s}=0$, where 
$s<\frac{n}{2}$. Thus $s<r$. Let $t$ be such that $t-s+n-r=\left[\left[\frac{n}{2}\right]\right]+1$. Let

$$
w=\left(x_{1}, \ldots, x_{s}, w_{s+1} \stackrel{t-s}{ }, \ldots, w_{t}, y_{t+1}, \ldots, y_{r}, y_{r+1}, \ldots, y_{n}\right)
$$

where $x_{s+1}>w_{s+1} \geq 0, \ldots, x_{t}>w_{t} \geq 0$. Then $t-s+n-r$ of the components of $x$ are strictly greater than the corresponding components of $w$. Thus $x P w$. Now $t-s$ components of $y$ are greater than the corresponding components of $w$ and $t-s \leq\left[\left[\frac{n}{2}\right]\right]$. Hence not $y P w$

Proposition 9 Let $x \in X$. Suppose $x$ is $C$-uncovered. Then either $x \in P S_{N}(R)$ or $x \in\left\langle J_{\frac{n}{2}}\right\rangle$.

Proof. Suppose $x \notin P S_{N}(R)$. Then $y \in X$ such that $y_{i}>x_{i}$ for some $i \in N$ and $\nexists j \in N$ such that $x_{j}>y_{j}$, where $x=\left(x_{1}, \ldots, x_{n}\right)$ and $\left(y_{1}, \ldots, y_{n}\right)$. Thus $y>x$. Hence $y R x$. Also $\forall z \in X, x P z \Rightarrow y P z$. We may assume without loss of generality that $x_{1} \leq y_{1}, \ldots, x_{r} \leq y_{r}, x_{r+1}<y_{r+1}, \ldots, x_{n}<y_{n}$ and $1 \leq n-r \leq$ $\left[\left[\frac{n}{2}\right]\right]$. If for $n / 2 \leq s \leq r, x_{1}=\ldots=x_{s}=0$, then $x \in\left\langle J_{\frac{n}{2}}\right\rangle$ since $r \geq n / 2$ $\left(r \geq n-\left[\left[\frac{n}{2}\right]\right]=\left\{\begin{array}{c}\left.\frac{n}{2} \text { if } n \text { is even, }\right) \text {. Suppose } x_{1}=\ldots=x_{s}=0, \text { where } \\ {\left[\left[\frac{n}{2}\right]\right]+1 \text { if } n \text { is odd. }}\end{array}\right.\right.$ $s<n / 2$. Then $\exists x_{s+1}^{\prime}, \ldots, x_{r}^{\prime}$ such that $0 \leq x_{i}^{\prime}<x_{i} i=s+1, \ldots, r$. Let $w=$ $\left(0, \ldots, 0, x_{s+1}^{\prime}, \ldots, x_{r}^{\prime}, x_{r+1}, \ldots, x_{n}\right)$. Then $y P w$ since $n-s$ of the components of $y$ are strictly greater than the corresponding components of $w$ and $n-s>n / 2$ since $s<n / 2$. Now not $x P w$ since $r-s$ of the components of $x$ are greater than 
the corresponding components of $w$ and $r-s \leq n / 2$ since $n / 2 \leq s \leq r$ whence $n-s \leq n / 2$. Thus $y C x$, contradicting the hypothesis that $x$ is $C$-uncovered.

Theorem 10 Suppose $x \notin\left\langle J_{\frac{n}{2}}\right\rangle$. Let $x \in X$. If $x$ is $C$-uncovered, then $x \in$ $P S_{N}(R)$.

Proof. Suppose $x \notin P S_{N}(R)$. Then $x \in\left\langle J_{\frac{n}{2}}\right\rangle$ by proposition 6 . Hence, $W^{-1}(x)=$ $\emptyset$. Let $y \in X-\left\langle J_{\frac{n}{2}}\right\rangle$. Then $W^{-1}(x) \subset W^{-1}(y)$ since $(0, \ldots, 0) \in W^{-1}(y)$. Clearly $y R x$. Thus $y C x$, a contradiction of the fact that $x$ is $C$-uncovered. Hence, $x \in P S_{N}(R)$.

Note that if $x \in\left\langle J_{\frac{n}{2}}\right\rangle$, then every element of $X$ is $C$-uncovered.

In words, all elements in the uncovered set are either in the Pareto set or they are in a special set of alternatives, $\left\langle J_{\frac{n}{2}}\right\rangle$. At first blush, the latter possibility appears to put a severe constraint on the ability of the definition of the covering relationship developed in this paper to result in a reasonably constructed uncovered set. Closer scrutiny, reveals the exception to be relatively mild in its effect. $J_{\frac{n}{2}}$ comprises all those alternatives that less than half of the players consider to be perfectly in the set of ideal points $(\alpha=1)$ and all remaining players consider entirely not in the set of ideal points $(\alpha=0) . \quad\left\langle J_{\frac{n}{2}}\right\rangle$ is the set of all alternatives that descend directly from those in $J_{\frac{n}{2}}$. By definition, none of the alternatives in either $J_{\frac{n}{2}}$ or $\left\langle J_{\frac{n}{2}}\right\rangle$ can defeat any alternative by majority vote. Hence, they can not cover any other alternative. Moreover, if there is even one alternative for 
which a majority of players express the slightest degree of preference (that is, a majority prefer the alternative at $\alpha>0$ ), then every alternative in $\left\langle J_{\frac{n}{2}}\right\rangle$ is covered by that alternative and by definition can not be in the uncovered set. While the alternative may tie elements in $\left\langle J_{\frac{n}{2}}\right\rangle$, it can defeat all alternatives lying in the region outside of the support for all players preferences (that is, all players preferences for these alternatives are $\alpha=0)$. Hence, it covers elements in $\left\langle J_{\frac{n}{2}}\right\rangle$. Thus, elements in $\left\langle J_{\frac{n}{2}}\right\rangle$ are uncovered if and only if $\left\langle J_{\frac{n}{2}}\right\rangle$ are the only elements in $X$. That is, no alternatives are supported by a majority at any $\alpha$-level.

\section{Conclusions and Some Final Considerations}

Existing definitions of the covering relation return different uncovered sets under thick indifference. Fuzzy preferences are thick. Thus, we have developed an appropriate definition of the covering relation for fuzzy preferences. Furthermore, we have demonstrated that in the absence of a maximal set, the resulting uncovered set is contained in the Pareto set. The only exception are those elements that are not strictly preferred by majority rule to at least one other alternative. Thus, as long as the entire set of alternatives is not confined to those elements, then all alternatives in the uncovered set are also in the Pareto set.

We return to the representation of the preferences of three players in figure 1 at the beginning of this paper. The maximal set is empty. There are six alternatives in the Pareto set: 


$$
P S_{N}(R)=\{(1,0,0),(0,1,0),(0,0,1),(.5, .25,0),(.25,0, .5),(0, .5, .25)\}
$$

The first three alternatives are elements of $\left\langle J_{\frac{n}{2}}\right\rangle$ and are not strictly preferred to any other alternatives. Given the results of this paper, the remaining three alternatives comprise the unique uncovered set:

$$
U C(X)=\{(.5, .25,0),(.25,0, .5),(0, .5, .25)\} .
$$

The model predicts that strategic players in an agenda amendment will pick one of these three alternatives.

However, our conclusion rests on the assumption that $\left\langle P S_{N}(R)\right\rangle$ denotes the set of alternatives. In other words, there is an alternative for every possible $n$-tuple of preferences descending from the Pareto set. As it turns out, $U C(X)$ can result in a non-Pareto efficient outcome when immediate predecessors of elements of the Pareto set $P S_{N}(R)$ are not contained in $X$, the set of alternatives, a situation we label "vulnerability to holes." We give consideration to this possibility before concluding.

Definition 11 Let $x, y \in X$. Suppose $x C y$. Let $W_{x, y}=\{w \in X \mid x P w$ and not $y P w, y \neq w \neq(0,0,0)\}$. Then $(x, y)$ is said to be vulnerable to holes if not $x C y$ 
in $X \backslash W_{x, y}$.

Proposition 12 Let $x, y \in X$. Suppose $x C y$. Then $x \notin\left\langle I_{1}\right\rangle$.

Proof. Suppose $x \in\left\langle I_{1}\right\rangle$. Then there does not exist $w \in X$ such that $x P w$. Hence not $x C y$.

Proposition 13 Let $x, y \in X$. Suppose $x C y$. Then $(x, y)$ is vulnerable to holes if and only if $y \notin\left\langle I_{1}\right\rangle$ and not $x P y$.

Proof. Suppose $(x, y)$ is vulnerable to holes. Suppose $y \in\left\langle I_{1}\right\rangle$. Then $x C y$ in $X \backslash W_{x, y}$ since $(0,0,0) \in W_{x, y}$ and not $y P(0,0,0)$, where the latter condition holds since $x \notin\left\langle I_{1}\right\rangle$ by the previous Proposition. It is not the case that $x P y$ else $x P y$ in $W_{x . y}$ since $y \in W_{x, y}$ and not $y P y$, i.e., $y$ is a $w$.

Conversely, suppose $y \notin\left\langle I_{1}\right\rangle$ and not $x P y$. Suppose $x C y$ in $W_{x, y}$. Then there exists $w \in X \backslash W_{x, y}$ such that $x P w$ and not $y P w$. Hence either $w=(0,0,0)$ or $w=y$. Suppose $w=(0,0,0)$. Since not $y P w, y \in\left\langle I_{1}\right\rangle$, a contradiction. Suppose $w=y$. Then $x P y$, a contradiction. Hence not $x C y$ in $X \backslash W_{x, y}$.

Theorem 14 Let $x, y \in X$. Suppose $x C y$. Suppose also that $(x, y)$ is vulnerable to holes. Then $\forall w \in W_{x, y}$, the following conditions hold:

(1) If $x \ngtr w$, then there exists a permutation $\pi$ of $\{1,2,3\}$ such that $x_{\pi(1)}=$ $y_{\pi(1)}>w_{\pi(1)}, w_{\pi(2)}>x_{\pi(2)}=y_{\pi(2)}$, and $x_{\pi(3)}>w_{\pi(3)} \geq y_{\pi(3)}$.

(2) If $x>w$, then $(a)$ there exists a permutation $\pi$ of $\{1,2,3\}$ such that $x_{\pi(1)}=$ $y_{\pi(1)}>w_{\pi(1)}, x_{\pi(2)}=y_{\pi(2)}=w_{\pi(2)}$, and $x_{\pi(3)}>y_{\pi(3)}=w_{\pi(3)}$ or $(b)$ there exists 
a component of $w$ strictly greater than the corresponding component of $y$ and there exists a permutation $\pi$ of $\{1,2,3\}$ such that $x_{\pi(1)}=y_{\pi(1)}=w_{\pi(1)}, x_{\pi(2)}=y_{\pi(2)}>$ $w_{\pi(2)}$, and $x_{\pi(3)}>w_{\pi(3)}>y_{\pi(3)}$.

Conversely, if $w \in X$ and satisfies either (1) or (2), then $w \in W_{x, y}$.

Proof. Let $w \in W_{x, y}$. Then $x \neq w$ since $x P w$. Since $y \notin\left\langle I_{1}\right\rangle, x>y$ by Proposition 4. Since $x R y$ and not $x P y$, two of the components of $x$ equal the two corresponding components of $y$ and the remaining component of $x$ is greater than the corresponding component of $y$.

Suppose $x \ngtr w$. Since $x P w$, two of the components of $x$ are strictly greater than the corresponding components of $w$ and the remaining component of $w$ is strictly greater then the corresponding component of $x$. For simplicity and without loss of generality, we can write either $(i) x_{1}=y_{1}>w_{1}, x_{2}=y_{2}>w_{2}$ and $w_{3}>x_{3}>y_{3}$. or $(i i) x_{1}=y_{1}>w_{1}, w_{2}>x_{2}=y_{2}$, and $x_{3}>y_{3}, x_{3}>w_{3}$. However $(i)$ does not hold else $y P w$. Suppose (ii) holds. If $y_{3}>w_{3}$, then $y P w$, a contradiction. Thus $w_{3} \geq y_{3}$. Hence (1) holds.

Suppose $x>w$. Then as in the previous paragraph, we can write without loss of generality, $x_{1}=y_{1}>w_{1}, x_{2}=y_{2} \geq w_{2}$, and either $(i) y_{3} \geq w_{3}$ or $(i i) w_{3}>y_{3}$. Suppose $(i)$ holds. Then $x_{1}=y_{1}>w_{1}$ and since not $y P w, x_{2}=y_{2}=w_{2}$ and $x_{3}>y_{3}=w_{3}$. In this case, (2) holds. Suppose (ii) holds. Then $x_{1}=y_{1}=$ $w_{1}, x_{2}=y_{2}>w_{2}$, and $x_{3}>w_{3}>y_{3}$ since not $y P w$. In this case, (2) holds.

For the converse, the only possible way $w=(0,0,0)$ is if $(2 a)$ holds, but then 
$y \in\left\langle I_{1}\right\rangle$, a contradiction. Clearly $w \neq y$. It is easily verified that $x P w$ and not $y P w$.

The situation in which $x \ngtr w$ occurs is illustrated in figure 2 . In such cases, the $\alpha$-levels of a majority of players' preferences are identical over the policy space.

[Place Figure 2 here.]

Figure 2 depicts holes where $x \ngtr w$ for some $w \in W_{x, y}$. Players $A$ and $B$ have identical preferences. Let $x=(.25, .25, .25)$ and let $y=(.25, .25,0)$. It follows that

$W_{x, y}=\{(1,0,0),(0,1,0),(.75,0,0),(0, .75,0),(.5,0,0),(0, .5,0),(.25,0,0),(0,0, .25)\}$

$W_{x, y} \in X$, and $x$ does not cover $y$.

The situation in which $x>w$ occurs is illustrated in figure 3. In such cases, the $\alpha$-levels of one or more of the players' preferences encompass the same region for that player over at least part of the policy space.

[Place Figure 3 here.]

Figure 3 illustrates holes where $x>w$ for $\forall w \in W_{x, y}$. The borders of the $\alpha$-levels at .25 and .5 for player $A$ are congruent at important segments of the policy space. Let $x=(.5, .75,0)$ and let $y=(.25, .75,0)$. It follows that 


$$
W_{x, y}=\{(.25, .5,0),(.25, .25,0),(.25,0,0)\}
$$

$W_{x, y} \in X$, and $x$ does not cover $y$.

We have demonstrated both phenomena using a three-player game. While the simplicity of the three-player game commends it for depicting these situations, it also greatly overstates the likelihood of the occurrence of either. The situation where $x \ngtr w$ is a particularly extraordinarily unique circumstance that is not likely to occur While the situation where $x>w$ is more likely, the probability of its occurrence will dramatically decrease as the number of players increases. With $T^{5}$, a three player game has 125 possible alternatives, a four player game has 625 alternatives, and a five player game has 3, 125 alternatives. With a high enough $N$, thousands of alternatives with the right preferences descending from the Pareto set would need to be missing.

We conclude by noting that we know of no sustaining argument for a majority coalition to choose a Pareto deficient alternative. Therefore, even in those rare situations in which a non-Pareto element is identified in the $U C(X)$ of a fuzzy model, we would be inclined to exclude it from consideration. 


\section{References}

[1] I. Shapiro, D.P. Green, Pathologies of Rational Choice Theory: A Critique of Applications in Political Science, Yale University Press, New Haven, 1994.

[2] R.D. McKelvey, Intransitivities in multidimensional voting models and some implications for agenda control, J. Economic Theory 12 (1976) 472-482.

[3] C.R. Plott, A notion of equilibrium and its possibility under majority rule, American Economic Review 57 (1967) 787-806.

[4] G. Tullock, Gordon, Why so much stability? Public Choice 37 (1981) 189202.

[5] J. Sloss, Stable outcomes in majority rule voting games, Public Choice 15 (1973) 19-48.

[6] C.A. Tovey, The instability of instability, NPSOR-91-15, Naval Postgraduate School, Monterey, CA, 1991.

[7] W.V. Gehrlein, F. Valognes, Condorcet efficiency: a preference for indifference, Social Choice and Welfare 18 (2001) 193-205.

[8] L. Ehlers, S. Barberà, Free triples, large indifference classes and the majority rule, Cahiers de recherche 2007-02, Universite de Montreal, Departement de sciences economiques, 2007, available: http://ideas.repec.org/p/mtl/montde/200702.html

[9] D.H. Koehler, Convergence and restricted preference maximizing under sim- 
ple majority rule: results from a computer simulation of committee choice in twodimensional space, American Political Science Review 95 (2001) 155-167.

[10] T. Bräuninger, Stability in spatial voting games with restricted preference maximizing, J. Theoretical Politics 19 (2007) 173-191.

[11] T.D. Clark, J.M. Larson, J.N. Mordeson, J.D. Potter, M.J. Wierman, Applying Fuzzy Mathematics to Formal Models in Comparative Politics, SpringerVerlag, Berlin, 2008.

[12] N.R. Miller, A new solution for tournaments and majority voting: further graph-theoretical approaches to the theory of voting, American Journal Political Science 24 (1980) 68-96.

[13] K.A. Shepsle, B.R. Weingast, Uncovered sets and sophisticated voting outcomes with implications for agenda institutions, American Journal Political Science 28 (1984) 49-74.

[14] R.D. McKelvey, Covering, dominance, and institution-free properties of social choice, American Journal Political Science 30 (1986) 283-315.

[15] L.A. Zadeh, Fuzzy sets, Information Control 8 (1965) 338-353.

[16] N.R. Miller, In search of the uncovered set, Political Analysis 15 (2007) $21-45$.

[17] E.M. Penn, Alternate definitions of the uncovered set and their implications, Social Choice and Welfare 27 (2006) 83-87.

[18] N.R. Miller, Alternative definitions of the covering relation: an extended 
tour, unpub. ms.

[19] G.A. Bordes, M. LeBreton, M. Salles, Gillies and Miller's subrelations of a relation over an infinite set of alternatives: general results and applications to voting games, Mathematics Operations Research 17 (1992) 509-518.

[20] G.A. Bordes, On the possibility of reasonable consistent majoritarian choice: some positive results, J. Economic Theory 31 (1983) 122-132.

[21] D. Austen-Smith, J.S. Banks, Positive Political Theory II: Strategy and Structure, University of Michigan Press, Ann Arbor, 2005. 


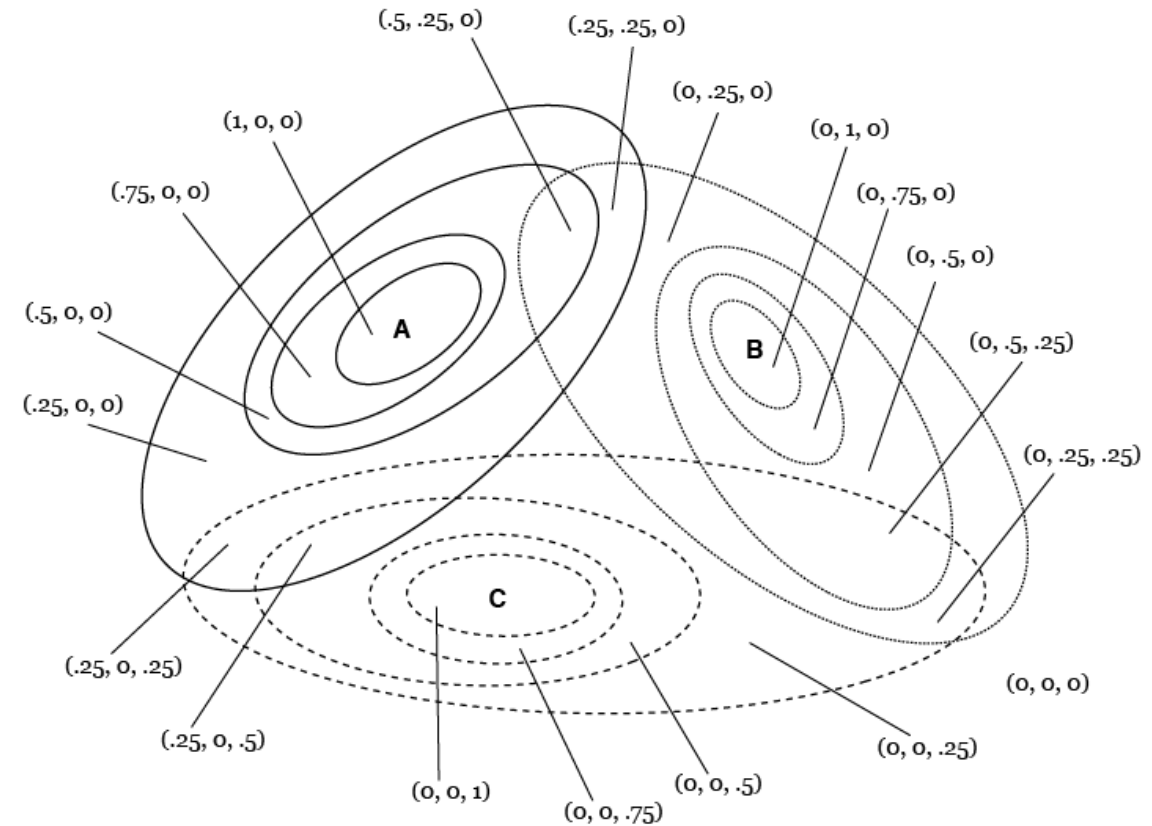

\begin{tabular}{|c|c|}
\hline Three-tuple & Winset \\
\hline$(1,0,0)$ & $(0, .5, .25),(0, .25, .25)$ \\
\hline$(.75,0,0)$ & $(0, .5, .25),(0, .25, .25)$ \\
\hline$(.5, .25,0)$ & $(0, .5,25)$ \\
\hline$(.5,0,0)$ & $(0, .5, .25),(0, .25, .25)$ \\
\hline$(.25, .250)$ & $(0, .5,25)$ \\
\hline$(.25,0,5)$ & $(.5,25,0)$ \\
\hline$(.25,0, .25)$ & $(.5,25,0)$ \\
\hline$(.25,0,0)$ & $(0, .5, .25),(0, .25, .25)$ \\
\hline$(0,1,0)$ & $(.25,0, .5),(.25,0, .25)$ \\
\hline$(0, .75,0)$ & $(.25,0, .5),(.25,0, .25)$ \\
\hline$(0, .5,25)$ & $(.25,0,5)$ \\
\hline$(0,5,0)$ & $(.25,0, .5),(.25,0, .25)$ \\
\hline$(0,25, .25)$ & $(.25,0, .5)$ \\
\hline$(0, .25,0)$ & $(.25,0, .5),(.25,0, .25)$ \\
\hline$(0,0,1)$ & $(.5, .25,0),(.25, .25,0)$ \\
\hline$(0,0,75)$ & $(.5, .25,0),(.25, .25,0)$ \\
\hline$(0,0, .5)$ & $(.5, .25,0),(.25, .25,0)$ \\
\hline$(0,0,25)$ & $(.5, .25,0),(.25, .25,0)$ \\
\hline$(0,0,0)$ & $\begin{array}{l}(.5,25,0),(.25, .25,0),(.25,0,5), \\
(.25,0,25),(0, .5, .25),(0, .25, .25)\end{array}$ \\
\hline
\end{tabular}

Figure 1: An Empty Maximal Set in a Fuzzy Spatial Model 


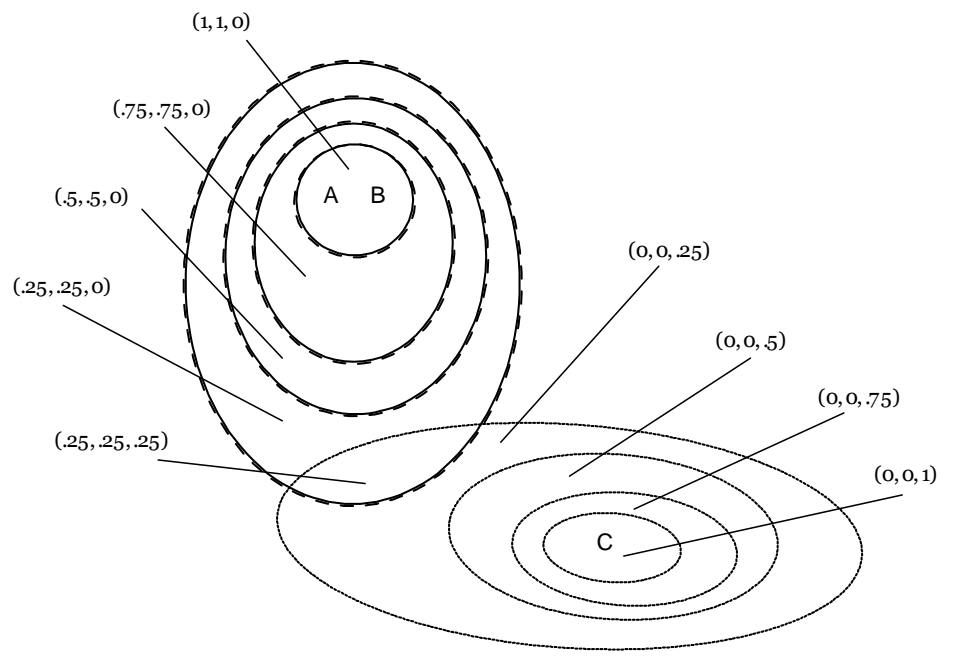

Figure 2: Players $A$ and $B$ with identical policy preferences. 


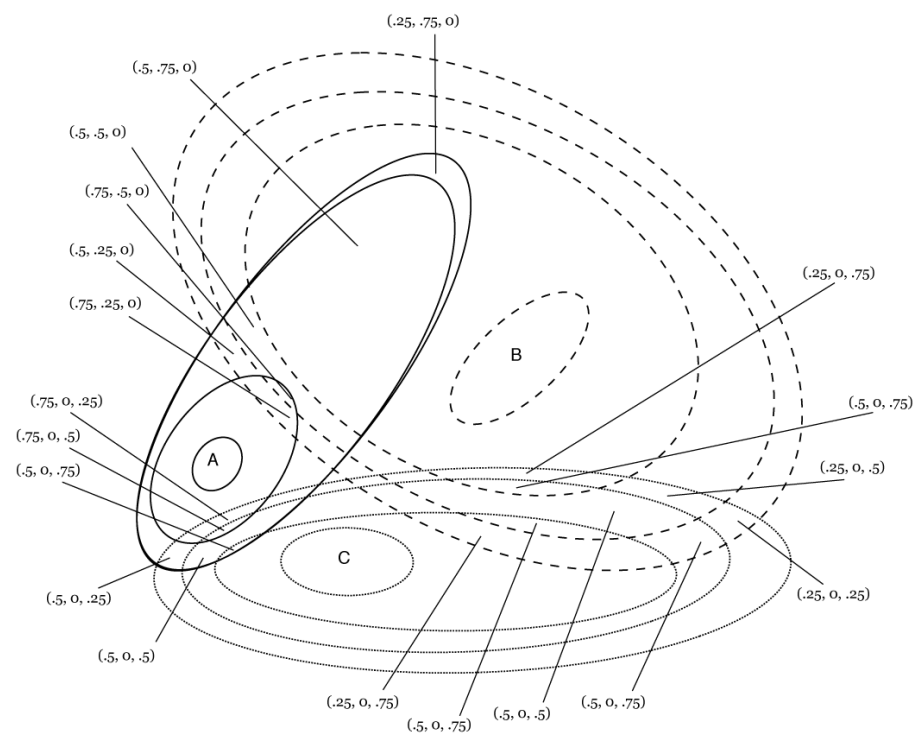

Figure 3: Player $A$ with congruent preferences at $\alpha=.25$ and .5 